Pramāna, Vol. 12, No. 4, April 1979, pp. 355-366, (C printed in India

\title{
On the holographic simulation of non-circular zone plates
}

\author{
S ANANDA RAO and S V PAPPU \\ Laboratories for Coherent Optics and Electro-Optical Research, \\ Department of Electrical Communication Engineering, \\ Indian Institute of Science, Bangalore 560012 \\ MS received 10 January 1979; revised 13 February 1979
}

\begin{abstract}
New methods involving the manipulation of fundamental wavefronts (e.g., plane and spherical) with simple optical components such as pinholes and spherical lenses have been developed for the fabrication of elliptic, hyperbolic and conical holographic zone plates. Also parabolic zone plates by holographic techniques have been obtained for the first time. The performance behaviour of these zone plates has been studied. Further a phenomenological explanation is offered for the observed improved fringe contrast obtained with a spherical reference wave.
\end{abstract}

Keywords. Holography; non-circular zone plates; fundamental wave fronts; optical components.

\section{Introduction}

Zone plate is a fascinating optical element and its potential applications are many and varied, since it combines the action of a lens as well as a diffraction grating. The early work about the optical behaviour of zone plates has been carried out about a century ago (Soret 1875; Cornu 1875). Since then a good amount of work on zone plates has been done and a fair amount of literature has been accumulated (Wood 1898; Myers 1951; Miyamoto 1961; Kock et al 1966; Hart et al 1966; Waldman 1966; Horman and Chau 1967; Stigliani et al 1967; Champagne 1968; Chau 1969; Dammann 1970; Rudolph and Schmahl 1970; Chang and George 1970; Moran 1971; Young 1971; Markus 1972; Barrett and Horrigan 1973; Kirz 1974).

A zone plate in its basic form consists of a series of alternate transparent and opaque zones on a suitable substrate. For example, the simplest way to make a zone plate is as follows. Concentric circles, with radii proportional to the square roots of whole numbers, are drawn on white paper. Alternate zones are then blackened and its photograph is taken. The transparency, when illuminated with a parallel beam of light, produces a large intensity at a point on its axis at a distance corresponding to the size of the zones and the wavelength of the light used. The photographic type zone plates could also be produced by taking advantage of the phenomenon of interference of light waves and zone plates thus obtained could be classified as regular interferometric zone plates (IZPs) or holographic zone plates (HZPs), depending upon the spatial resolution of the medium used for recording the interference fringes.

Of course, there is the mechanical way, analogous to the conventional way for 
making ruled diffraction gratings, for the fabrication of zone plates and they are designated as geometric zone plates (GZPs).

A perfect sinusoidal variation of intensity for the zones could be recorded in the case of HZPs, in view of the fact that in holography, unlike in conventional interferometry, one uses a photographic emulsion (or other suitable recording medium) of required high resolution and dynamic range (i.e., required linear portion of the $E-t$ curve) in order to faithfully record all the modulations of intensity available in the interfering wavefronts. Hence an HZP will not produce multiple diffraction orders; whereas a GZP or IZP would produce multiple orders in view of the fact that their zones possess square wave periodicity. The square wave periodicity in an IZP is a consequence of the use of low spatial resolution emulsion. In other words, the modulations available between $I_{\max }$ and $I_{\min }$ are recorded more or less in a binary fashion. Further an HZP will not produce ghost images as a GZP would and in view of all these advantages HZPs are becoming increasingly popular optical elements in a wide variety of optical systems. As a matter of fact, a hologram is nothing but a zone plate in action and this fact was demonstrated by Rogers (1950) on the heels of the discovery of holography by Gabor.

It is a common practice to designate a zone plate as circular or linear depending upon whether the zones are circular or straight. We can easily conceive of noncircular zone plates by following this conventional nomenclature. Thus zone plates containing elliptic zones will have to be designated as elliptic zone plates etc. Circular and linear zone plates are relatively easy to fabricate and hence their properties have been studied rather extensively. In recent years, it has been discovered that non-circular zone plates (e.g., elliptic, hyperbolic, etc.) do find applications in optical computing systems, for example, and hence some attempts have been made in the past to fabricate non-circular zone plates by employing holographic methods (Fredricks 1969, 1970; Iwasaki 1976; Aggarwal et al 1978). The major drawback of these methods is that the required object wavefront is derived by a complicated optical set-up involving the use of cylindrical/conical lenses.

It is found that the non-circular HZPs (viz., elliptic, hyperbolic and conical) could be produced relatively effortlessly by using a simple optical system as shown in figure 1 , wherein only the manipulation of fundamental wavefronts (viz., plane and spherical) by simple optical elements such as pinholes or simple spherical lenses is involved. As a matter of fact these fundamental wavefronts are referred to by Stroke (1969) as the building blocks of holography. Recently, we have utilised these fundamental wavefronts for the holographic simulation of a wide variety of mirrors (Ananda Rao and Pappu) and now we have used them for the generation of non-circular HZPs.

The purpose of this paper is to describe new and simple methods for the fabrication of several non-circular HZPs and discuss their performance. Further, an explanation is offered for the difference in fringe contrast observed between the utilisation of a spherical wave and a plane wave as a reference wave.

\section{Experimental}

The desired object and reference wavefronts are obtained by using the set-up shown in figure 1. We have used in our experiments a $0.5 \mathrm{~mW}$ Spectra Physics Model $155 \mathrm{He}$ Ne laser as the source and Agfa 10E75 photographic plates as the recording media. 


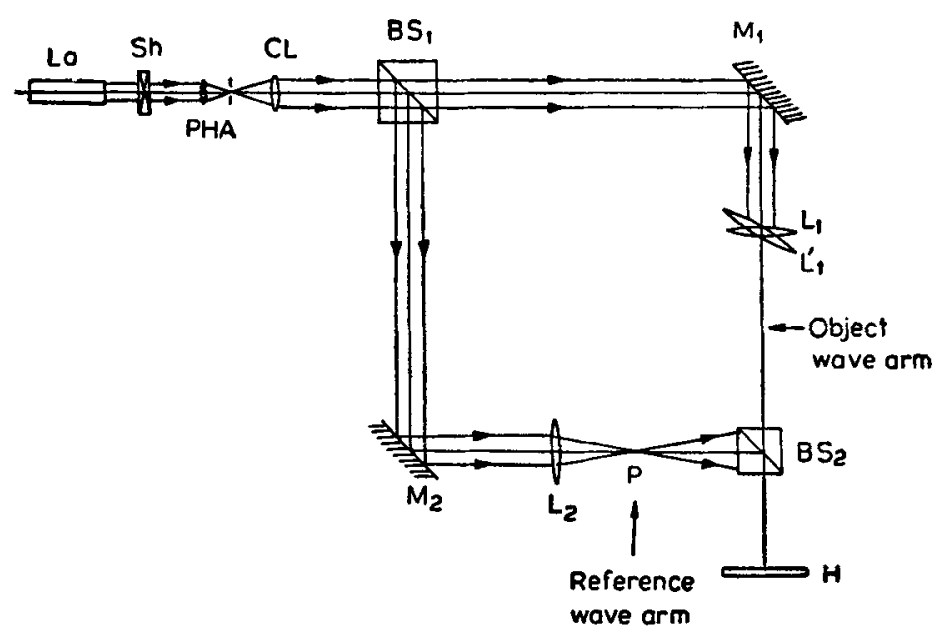

Figure 1. Schematic of the set-up used for the construction of various types of holographic zone plates (HZPs). La=laser, $\mathrm{Sh}=$ shutter, $\mathrm{PHA}=$ pinhole assembly, $\mathrm{CL}=$ collimating lens, $\mathrm{BS}_{1}$ and $\mathrm{BS}_{2}=$ beam splitters, $\mathrm{M}_{1}$ and $\mathrm{M}_{2}=$ mirrors, $\mathrm{L}_{1}=$ simple spherical lens in the object wave arm, $L_{1}^{\prime}=$ tilted position for the lens $L_{1}, L_{2}^{\prime}=$ simple spherical lens in the reference wave arm, $\mathbf{P}=$ point source derived with lens $\mathrm{L}_{2}, \mathrm{H}=$ holographic plate.

The salient features about the utilisation of the set-up shown in figure 1 are as follows. We could derive a plane or a spherical wave as reference wave just by removing or keeping in place the lens $\mathrm{L}_{2}$. Wtih lens $\mathrm{L}_{1}$ in place, we get the facility to mix a spherical object wave with either a plane or a spherical reference wave. Under these circumstances, circular fringes will be formed, which when recorded on plate $H$, will yield a circular zone plate.

The desired non-circular (e.g., elliptic and hyperbolic) object wavefronts could be generated effortlessly just by tilting the lens $L_{1}$ through a suitable angle as shown schematically by $L_{1}^{\prime}$ in figure 1 . These non-circular object wave surfaces could then be mixed with either a plane or a spherical reference wave and the resulting fringe system could be recorded on the photographic plate $H$. Upon processing, the plate will function as a particular type of zone plate (e.g., elliptic, hyperbolic, etc.) depending upon the nature of the contours of the fringes (viz., elliptic, hyperbolic, etc.) recorded on it.

The HZPs in our experiments have been realised by using the on-axis method of recording. The performance of the zone plates is checked under plane wave illumination. During this reconstruction schedule, however, a simple innovation of illuminating the HZP away from its central region has been incorporated and this has given the advantage of separating out the various images from the DC region. In other words, the benefits that would have accrued had we recorded the HZPs by the conventional Leith and Upatnieks off-axis method have been realised effortlessly by our simple trick.

\section{Results and discussion}

Before we proceed with the actual realisation of the desired non-circular HZPs, it is necessary to know the nature of the object wave surface emerging from the tilted lens 
in figure 1. It is discovered that the foolproof way of finding out the nature of the wave surface existing in space is to mix it with a plane reference wave and then make a record of the resulting fringe system. By this procedure, the output wave surfaces emerging from the tilted lens at various points of their propagation path have been identified to follow the sequence: ellipsoidal waves--vertical line focus-hyperboloidal waves-horizontal line focus-ellipsoidal waves again, as shown in figure 2.

With a spherical reference wave, however, it was not possible to ascertain unambiguously the nature of the wave surface existing in space, because the fringe system obtained in this case is found to be dependent upon the location of the point souree $P$ in figure 2 and thus the fringe system observed is sometimes different from those presented in the sequence shown in figure 2.

In the light of our observations, we conclude that a plane reference wave is ideally suited for an unambiguous identification of a wave surface existing in space. Hence a plane reference wave could be called as, to coin a phrase, an Analyser wave.

\subsection{Elliptic and hyperbolic HZPS}

The principles of operation of elliptic and hyperbolic zone plates are essentially the same, in that both give rise to a vertical and a horizontal focal lines. But there can be situations where an elliptic zone plate is to be preferred to a hyperbolic zone plate and vice-versa. Hence we have fabricated both elliptic and hyperbolic HZPs separately and checked their performance.

The elliptic HZPs are obtained by recording the elliptic fringes shown in figure 2 on a suitably located holographic plate. Similarly, hyperbolic HZPs are realised by recording the hyperbolic fringes shown in figure 2 . We have chosen to show, as an example, the results obtained with a hyperbolic HZP when it is illuminated with a plane wave and the results are shown in figure 3. The important features to note are the evolutions of the vertical line focus and the horizontal line focus through the intermediate stages of elliptic/hyperbolic waves. The noteworthy features of our results, in contrast to the results of earlier workers who have used the off-axis method for the realisation of zone plates (Aggarwal et al 1978), is the unambiguous revelation of the character of the wave surfaces that exist in space as a function of propagation distance during reconstruction. This revelation has been possible because of the interference effects between the DC component and the output wave.

It should be mentioned here that there is still simpler method for the realisation of elliptic HZPs. This involves the use of the set-up of figure 1 in the configuration for the realisation of a circular zone plate and then tilting the holographic plate $H$ suitably in order to record the elliptical fringes. The principle that the geometrical projection of a circle onto an inclined plane is an ellipse is exploited here. It should be obvious that hyperbolic HZPs cannot be produced with this method.

The imaging performance of a hyperbolic HZP has been studied using a transparency containing a triangle as the object. The images obtained are displayed in figure 4. Similar results are naturally to be expected from elliptic HZPs also and they have been obtained.

\subsection{Conical HZPS}

A conical HZP is realised by the following method. The lens $L_{1}$ in its normal 


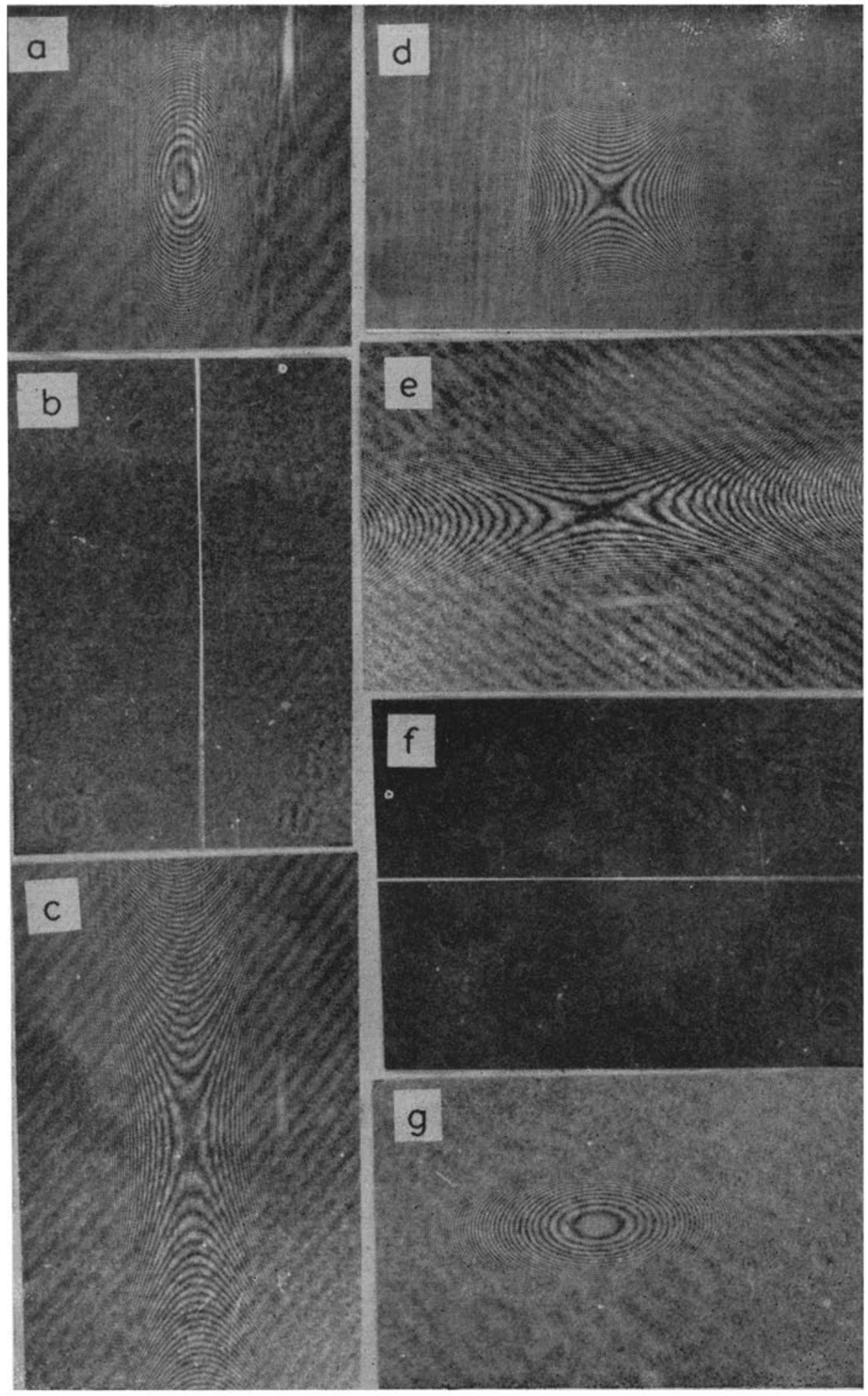

Figure 2. (See caption in p. 366) 


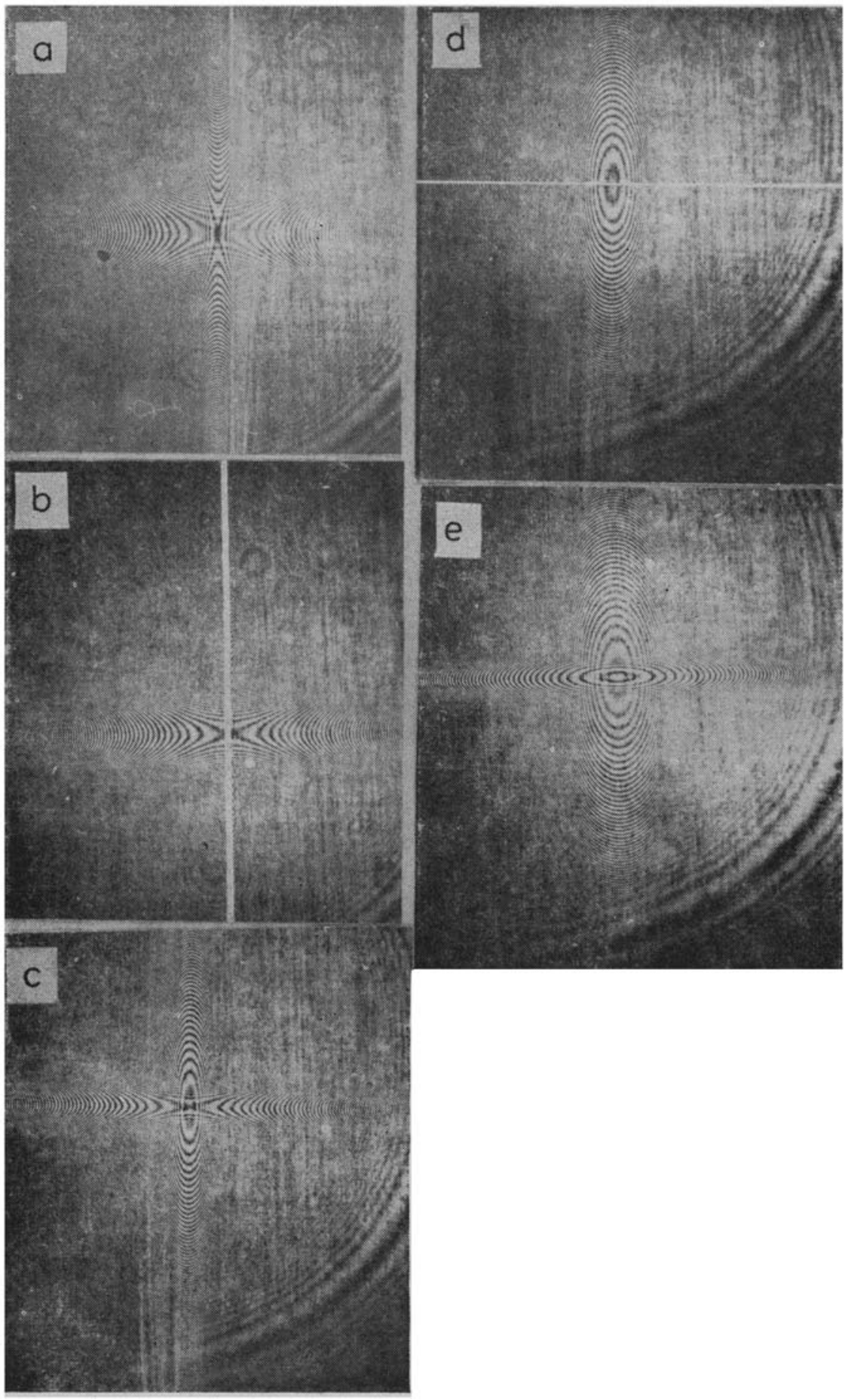

Figure 3. (a) to (e) represent the sequence of reconstructed wavesurfaces, as a function of propagation distance, obtained from a hyperbolic HZP upon its illumination with a plane wavefront. Since the HZPs are derived by employing the on-axis methods, the observed fringe system is to be expected because of the interference of the DC component with the output wave. Note the expected formation of the vertical and the horizontal focal lines respectively in figures $\mathbf{3}(\mathbf{b})$ and $\mathbf{3}(\mathbf{d})$. 

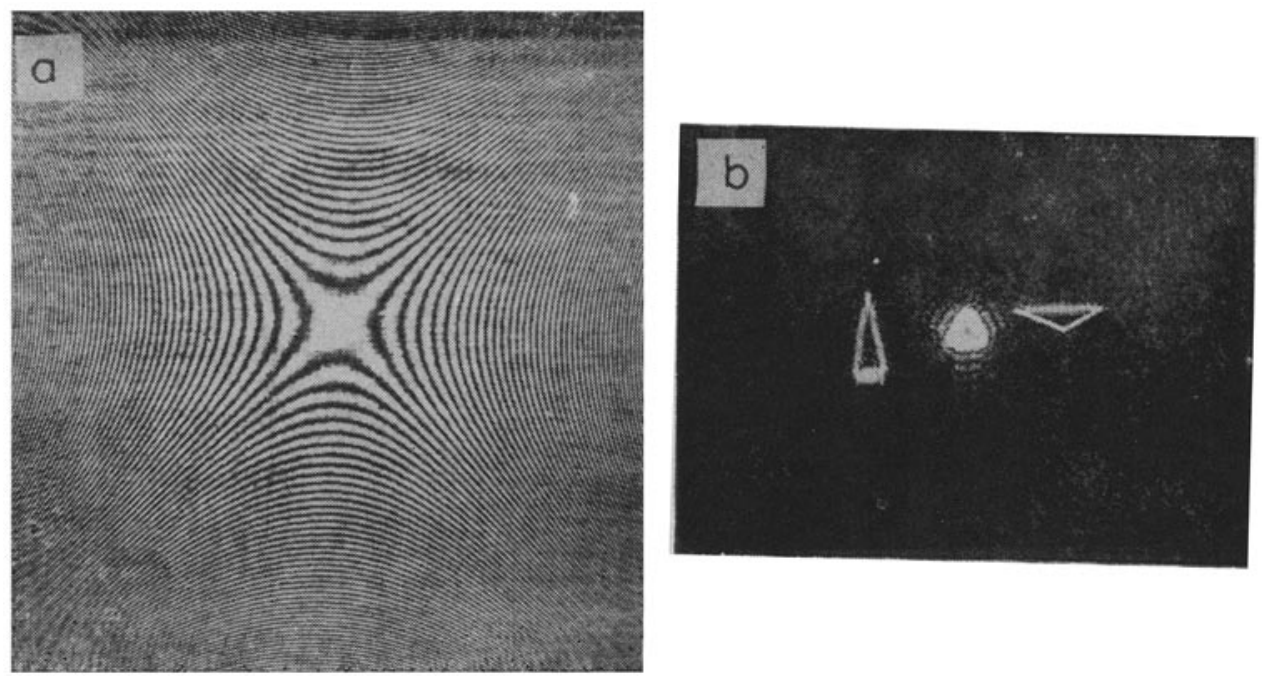

Figure 4.
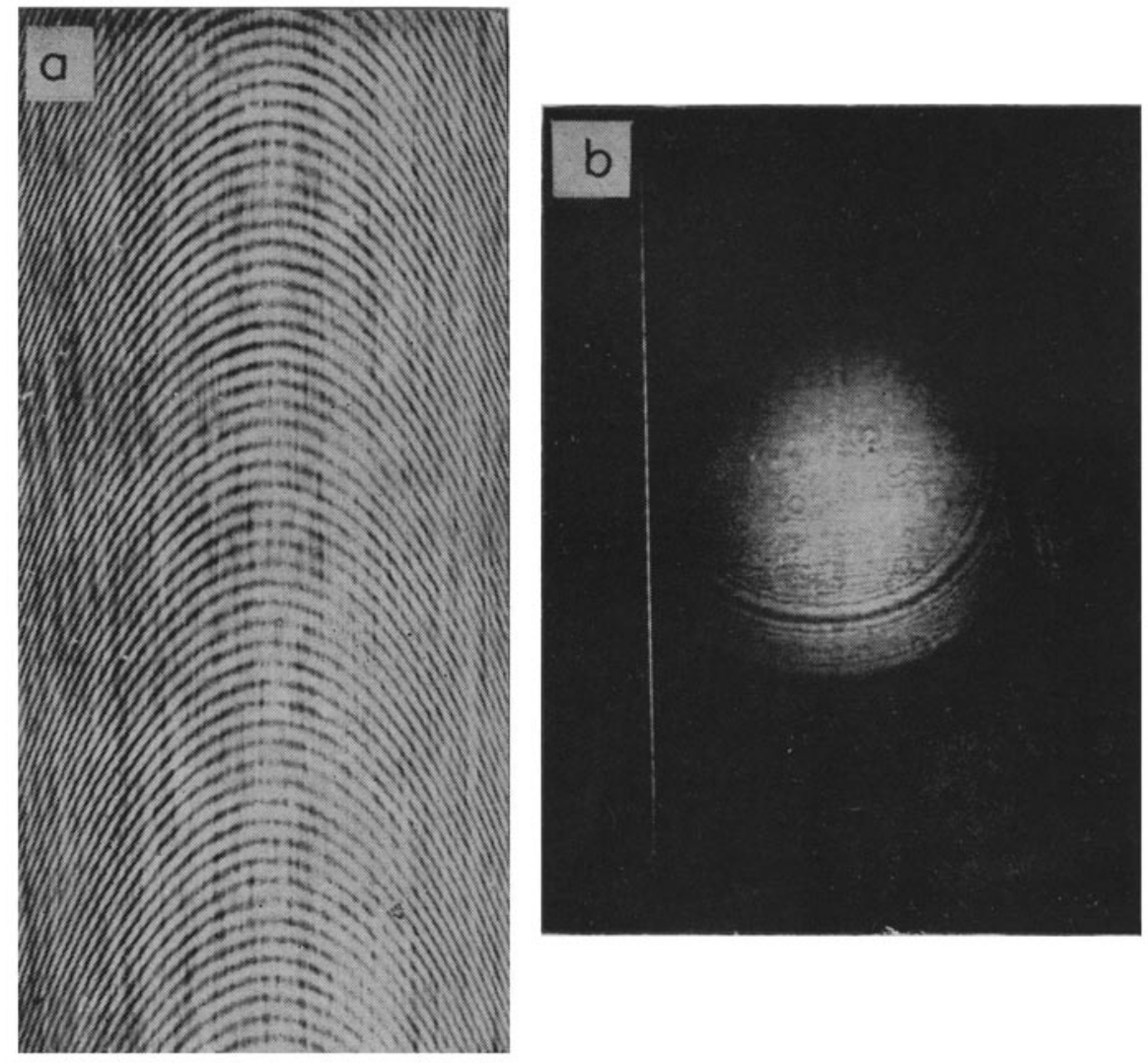

Figures 4 \& 5. (See captions in p. 366) 

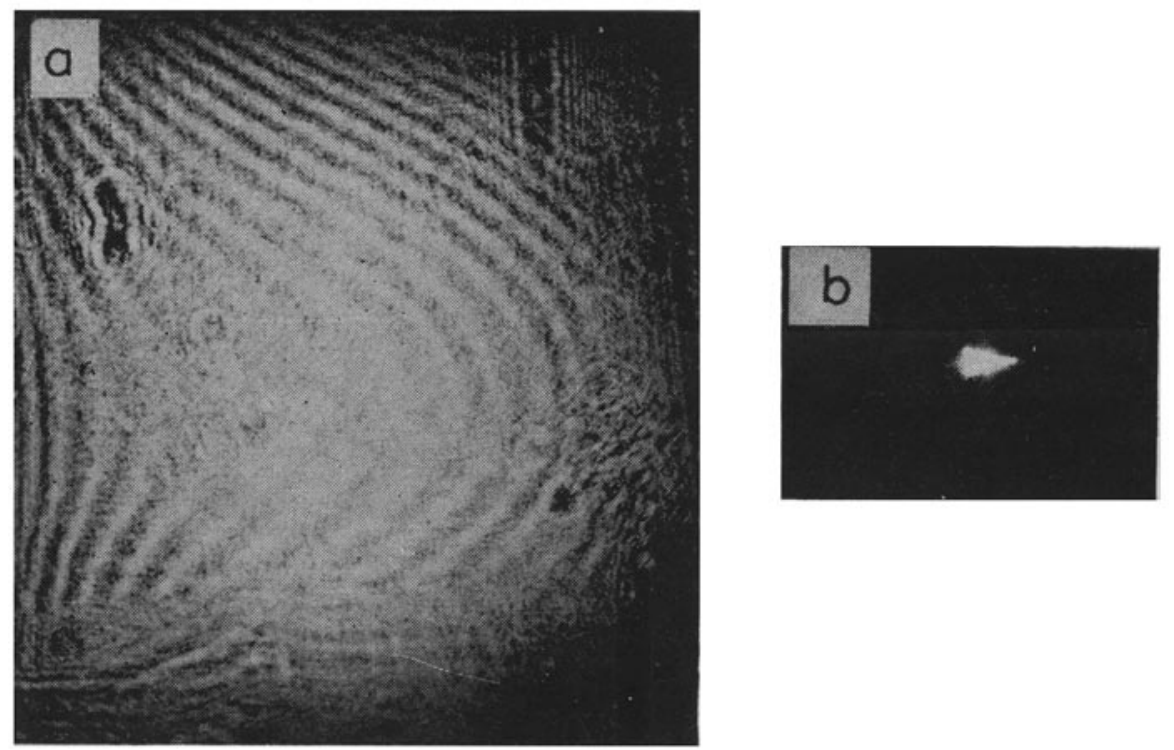

Figure 6. Performance of a Parabolic HZP. (a) Photograph of a parabolic HZP. (b) Line focus obtained in reconstruction from it which again is illuminated (to conform to the off-axis mode of reconstruction) with a plane wavefront. Here the DC is also shown. 
Table 1. Summary of the fringe system observed and the zone plate realised

\begin{tabular}{llll}
\hline \multicolumn{1}{c}{$\begin{array}{c}\text { Object } \\
\text { Wave }\end{array}$} & $\begin{array}{c}\text { Reference } \\
\text { Wave }\end{array}$ & $\begin{array}{l}\text { Fringe System } \\
\text { Observed }\end{array}$ & $\begin{array}{l}\text { Zone plate } \\
\text { Realised }\end{array}$ \\
\hline Elliptic & Spherical & $\begin{array}{l}\text { Elliptic or } \\
\text { hyperbolic }\end{array}$ & $\begin{array}{l}\text { Elliptic or } \\
\text { hyperbolic } \\
\text { Elliptic }\end{array}$ \\
Elliptic & Plane & Elliptic \\
Hyperbolic & Spherical & $\begin{array}{l}\text { Hyperbolic or } \\
\text { elliptic }\end{array}$ & $\begin{array}{l}\text { Hyperbolic } \\
\text { or elliptic } \\
\text { Hyperbolic } \\
\text { Conical }\end{array}$ \\
Hyperbolic & Plane & Hyperbolic & Conical \\
Conical & Spherical & Parabolic \\
Cylindrical & Spherical & Parabolic & \\
\hline
\end{tabular}

position is translated off-axis suitably such that a conical focus representing the state of maximal coma and minimal astigmatism (Kingslake 1927; Ose et al 1971) is obtained as its output. The wavefront emerging from such a focus is mixed with a spherical reference wave and a record of the resultant fringe contours has been made. The actual photograph of a conical HZP and the focus obtained from it during illumination with a plane wave are shown in figure 5.

\subsection{Parabolic HZPS}

To the best of our knowledge no attempt has been made in the past to fabricate a parabolic zone plate. We have been able to realise a parabolic HZP and in order to get it we had to replace the spherical lens $\mathrm{L}_{1}$ with a cylindrical lens. The cylindrical wavefront is then mixed with a spherical reference wavefront suitably. The photography of a parabolic HZP and the results obtained from it upon its illumination with a plane wavefront are shown in figure 6 .

A point worth mentioning here is about the sharpness of the line focus obtained with these parabolic HZPs. As a matter of fact, this is to be expected since a parabolic mirror gives rise to the sharpest focal spot and in the case of a parabolic HZP the line focus is due to the juxtaposition of a number of sharpest focal spots.

A summary of the fringe system observed and the nature of the zone plates realised under the influence of spherical/plane reference waves is given in table 1 .

\section{Fringe contrast improvement}

In all our experiments, we have observed that the fringe contrast is better with a spherical reference, wave than with a plane reference wave. Plane and spherical waves have been used extensively in holographic work; but it appears no one has paid attention to notice and to explain the better fringe contrast obtained with spherical reference wavefronts. A simple analysis shows that what we have observed should be the case and the analysis is given below.

For simplicity let us consider that a spherical object wave given by

$$
S_{0}=\left(A_{0} / Z_{0}\right) \exp \left(j k Z_{0}\right)
$$


is mixed with either a plane reference wave given by

$$
S_{r}=A_{r} \exp j\left(k Z_{r}+\phi\right)
$$

or a spherical reference wave given by

$$
S_{r}=\left(A_{r} / Z_{r}\right) \exp j\left(k Z_{r}+\phi\right)
$$

where $A_{0}, A_{r}, k$ and $\phi$ have the usual meaning. $Z_{0}$ and $Z_{r}$ represent the distances of the object and the reference source respectively from the holographic plate. The fringe contrast when the spherical object wave is mixed with a spherical reference wave can be expressed as (Pernick and Leib 1978)

$$
C_{s s}=\frac{2 A_{0} A_{r} Z_{0} Z_{r}}{A_{0}^{2} Z_{r}^{2}+A_{r}^{2} Z_{0}^{2}}
$$

The fringe contrast when the spherical object wave is made to interfere with a plane reference beam is given by,

$$
C_{s p}=\frac{2 A_{0} A_{r} Z_{0}}{A_{0}^{2}+A_{r}^{2} Z_{0}^{2}}
$$

Our experimental observation suggests that $C_{s s}>C_{s p}$, which means that

$$
C_{s s}-C_{s p}=\left(Z_{r}-1\right)\left(A_{r}^{2} Z_{0}^{2}-A_{0}^{2} Z_{r}\right),
$$

must be positive. Since $Z_{r}$ and $Z_{0}$ are always greater than unity, it means that

$$
\left(A_{r}^{2} Z_{0}^{2}-A_{0}^{2} Z_{r}\right)>0
$$

It is a common practice in holography to use a reference beam whose strength is greater than the object beam. This means $A_{r}>A_{0}$ and with this condition then (3) conveys that $C_{s s}-C_{s p}$ would always be positive if $Z_{0}>Z_{r}$. This condition is invariably fulfilled in all holographic work and hence it can be concluded that a better fringe contrast should naturally be expected in the case of a spherical reference beam, as we have observed in all our experiments.

\section{Conclusions}

Simple methods for the fabrication of elliptic, hyperbolic, conical and parabolic holographic zone plates are described and the performance of these zone plates has been studied. The basic methodology for the simulation of the noncircular zone plates involves the manipulation of fundamental wavefronts (viz., plane and spherical) with simple optical elements such as pinholes and spherical lenses.

It is observed that the fringe contrast is always superior in the case of a spherical reference wave and a simple phenomenological explanation for this observation is offered. 


\section{Acknowledgement}

One of the authors (SAR) acknowledges the support from the Quality Improvement Program (QIP), Ministry of Education, Government of India, for pursuing his studies at the Institute leading to $\mathrm{a} \mathrm{PhD}$ degree.

\section{References}

Aggarwal A K, Gupta P C, Mullick S K and Subramanian P 1978 Appl. Opt. 17987

Ananda Rao $S$ and Pappu S V (to be published)

Barrett H and Horrigan F 1973 Appl. Opt. 122686

Champagne E 1968 Appl. Opt. 7381

Chang M and George N 1970 Appl. Opt. 9713

Chau H H M 1969 Appl. Opt. 81209

Cornu A (Poggendorff; 1875) Ann. Phys. (Leipzig) 156114

Dammann H 1970 Optik 3195

Fredricks R J 1969 SPIE J. 7106

Fredricks R J 1970 The University of Michigan Ph.D. Thesis 71-406 University Microfilms (Ann Arbor, Michigan)

Hart H E, Scrandis J B and Mark R 1966 J. Opt. Soc. Am. 561018

Horman M H and Chau H H M 1967 Appl. Opt. 6 317, 1415

Iwasaki T 1976 J. Opt. Soc. Am. 66277

Kingslake R 1927 Trans. Opt. Soc. (London) 2794

Kirz J 1974 J. Opt. Soc. Am. 64301

Kock W E, Rosen L and Rendeiro J 1966 Proc. IEEE 541599

Markus F A 1972 Opt. Spectrosc. 32661 (English Transl. of Optik Spektrosk.)

Miyamoto K 1961 J. Opt. Soc. Am. 5117

Moran J M 1971 Appl. Opt. 10412

Myers Ora E Jr 1951 Am. J. Phys. 19359

Ose T, Noguchi M and Kubota T 1971 Applications of holography (New York: Plenum Press) p 62

Pernick B J and Leib K G 1978 Am. J. Phys. 4690

Rogers G L 1950 Nature (London) 166237

Rudolph D and Schmahl G 1970 Optik 30475

Soret J L (Poggendorff; 1875) Ann. Phys. (Leipzig) 15699

Stigliani D, Mittra R and Semonin R G 1967 J. Opt. Soc. Am. 57610

Stroke G W 1969 An introduction to coherent optics and holography (New York: Academic Press) p 12

Waldman G 1966 J. Opt. Soc. Am. 56215

Wood R W 1898 Philos. Mag. 45511

Young M 1971 J. Opt. Soc. Am. 62972 
Figure 2. Sequence of output wave surfaces emerging from the lens $L_{1}$ when tilted about its vertical axis, as a function of propagation distance. The fringe system shown here is obtained by mixing a plane wavefront with the corresponding wave surface. (a) Vertical elliptic fringe system corresponding to the defocused state before the vertical line focus. (b) Vertical line focus. (c) Vertical hyperbolic fringe system corresponding to the defocused state after the vertical line focus. (d) Hyperbolic fringe system with equal eccentricities for its two branches corresponding to the midway location between the vertical and the horizontal line foci. (e) Horizontal hyperbolic fringe system corresponding to the defocused state before the horizontal line focus. (f) Horizontal line focus. (g) Horizontal elliptic fringe system corresponding to the defocused state after the horizontal focal line. It should be noted that no fringes will appear in figures $2(\mathbf{a}),(\mathbf{c}),(\mathbf{d}),(\mathbf{e})$ and $(\mathrm{g})$ had we not mixed the wave surfaces with a plane reference wavefront. The fringe visibility in figures 2(b) and $2(\mathbf{f})$ is poor because of $!$ he overwhelming intensity of the object wave appearing as focused energy. Another point to be noted is the fact that the sequence will go from (g) to (a) if the lens $L_{1}$ is tilted about the horizontal axis. The background fringes in all the photographs are due to noise.

Figure 4. Imaging pcrformanee of a hyperbolic HZP. The zone plate is realised in the on-axis mode. But its imaging behaviour is checked by illuminating the zone plate on its side (i.e., away from the central region) with a wavefront coming from the object transparency which is illuminated with a plane wavefront. This in effect means an off-axis mode of reconstruction from an on-axis zone plate and hence the images due to the vertical focusing and the horizontal focusing actions of the hyperbolic HZP will be separated from the DC. (a) Photograph of the hyperbolic HZP. (b) Images of an object transparency as obtained with the hyperbolic HZP. The object, namely the equilateral triangle, can be seen in the DC region, while the images due to the vertical focusing action and the horizontal focusing action of the HZP could be seen to the left and the right sides of the DC respectively.

Figure 5. Performance of a conical HZP. (a) Photograph of the conical HZP. (b) Conical focus obtained in reconstruction from the HZP upon its illumination with a plane wavefront. Here also, as in the case of hyperbolic HZP, the reconstruction is achieved by illuminating the conical HZP to correspond to an off-axis mode of reconstruction. Hence the conical focus (shown in the figure) is separated from the DC (not shown). 